Disclosure of Interest: None declared

DOI: 10.1136/annrheumdis-2017-eular.6326

\section{THU0009 IN VITRO STUDIES USING CYBRIDS, SHOW THAT MTDNA FROM HEALTHY AND OA PATIENTS HAVE DIFFERENT MITOCHONDRIAL ACTIVITY}

M. Fernández-Moreno ${ }^{1,2}$, A. Dalmao-Fernandez ${ }^{2}$, T. Hermida-Gomez ${ }^{2}$, M.J. Sanchez-Dopico ${ }^{2}$, M.E. Vazquez-Mosquera ${ }^{2}$, E. Cortes-Pereira ${ }^{2}$, S. Relaño-Fernandez ${ }^{2}$, I. Rego-Perez ${ }^{2}$, F.J. Blanco ${ }^{2} .{ }^{1}$ Centro de Investigación Biomédica en Red, Ciber-Bbm, Madrid; ' 2 Servicio de Reumatología. Complexo Hospitalario Universitario (CHUAC), Instituto de Investigación Biomédica de A Coruña (INIBIC), A Coruña, Spain

Background: Mitochondrial dysfunction is well documented in OA and has the capacity to promote abnormalities in chondrocyte function and viability contributing to cartilage degeneration. Previous studies have showed the mtDNA haplogroup $\mathrm{J}$ are associated with incidence and progression of $\mathrm{OA}$. Cybrids are optimal cellular models to study the real mitochondrial biology and function implications in the cellular behaviour, since they carry different mitochondrial variants with the same nuclear background, therefore, excluding the variations because of nuclear genome.

Objectives: The aim of this work is to test the real role of mtDNA in cellular activity, using cybrids with mtDNA from healthy donors (without $O A$ ) and from patients with knee OA.

Methods: Cybrids were developed using 143B.TK- Rho-0 cell line (nuclear donor) and platelets (mitochondrial donors) from healthy (without $\mathrm{AO}-\mathrm{N}-$ ) and knee OA donors. The OXPHOS function was evaluated by SeaHorse XFp after addition of oligomycin, FCCP and Rotenone/Antimycin A. The metabolic status was evaluated by glucose consumption and lactic acid production. The glycolytic activity was measure after addition of glucose, oligomycin and 2-dioxyglucose using Seahorse XFp. Appropriate statistical analyses were performed with GraphPad Prism v6.

Results: OA cybrids had lower basal respiration $(92.07 \pm 39.9$ and $155.5 \pm 54.08$, $p<0.0005)$, and maximal respiratory capacity $(114.7 \pm 50.1$ and $160.6 \pm 44.7$, $\mathrm{p}<0.05$ ) than $\mathrm{N}$. The analysis of ATP production was lower in OA than in N cybrids $(66.69 \pm 28.69$ versus $101.5 \pm 42.04 \mathrm{p} \leq 0.05)$. The $\%$ spare respiratory capacity value for the $\mathrm{N}$ was significantly lower than in $\mathrm{OA}$ cybrids $(107 \pm 16.21$ versus 124.7 $\pm 5.97, p<0.0005)$. Cybrids carrying the mtDNA from OA patients showed higher glucose consumption than $\mathrm{N}$ cybrids $(43.77 \pm 8.87 \mathrm{mg} / \mathrm{ml}$ and $31.91 \pm 13.69$ $\mathrm{mg} / \mathrm{ml} ; \mathrm{p}<0.05$ ) however in the lactic acid production did not exit differences. The glycolytic activity was evaluated and showed that OA cybrids had lower glycolysis $(71.05 \pm 4.83$ versus $85.43 \pm 11.18, \mathrm{p}<0.05)$ but higher glycolytic reserve than $\mathrm{N}$ cybrids $(56.60 \pm 7.5$ versus $39.73 \pm 19.13, p \leq 0.05)$.

Conclusions: Cybrids have different metabolic behaviour, being $\mathrm{N}$ more efficient using glucose via glycolysis. We found differences statistical significate in the parameters that describe the mitochondrial respiration capacity, in this line OA cybrids had lower mitochondrial respiration and produce less ATP than the cybrids obtained from healthy patients. These results showed that the mitochondria obtained from healthy and OA donors had a different behaviour. These data also offer a real rationale for why mitochondria alterations play an important role in the incidence of OA.

Disclosure of Interest: None declared

DOI: 10.1136/annrheumdis-2017-eular.5930

\section{THU0010 ALTERED MIRNAS PROFILES IN PLASMA-DERIVED EXOSOME OF PATIENTS WITH ANKYLOSING SPONDYLITIS BY SMALL RNA-SEQ ANALYSIS}

Y. Huang, T. Li, Z. Huang, W. Deng, S. Zheng, X. Guo, Z. Huang. Guangdong No. 2 Provincial People's Hospital, Guangzhou, China

Background: Ankylosing spondylitis (AS) is a chronic inflammatory disease, which is difficult to diagnose in the early stages. Increasing evidences have shown that MicroRNAs (miRNAs) may serve as novel biomarkers for AS. Exosome can function as vehicles to deliver miRNAs in body fluids including saliva and plasma. However, the relationship between exosome-delivered miRNAs and AS has yet to be determined.

Objectives: The aim of this study is to detect the altered miRNAs profiles of plasma-derived exosome in AS patients by small RNA-Seq Analysis.

Methods: Ribo ${ }^{\mathrm{TM}}$ kit was used to isolate exosome. Small RNA Sample Pre Kit was used to build libraries in 3 AS patients and 3 healthy volunteers (HV), following by IlluminaHiSeq platform sequencing and bioinformatics analysis. Quantitative reverse-transcription PCR (qRT-PCR) was used to confirm the expression of the highly-expressed miRNA in another $10 \mathrm{AS}$ patients and $10 \mathrm{HV}$, and receiveroperator characteristic $(\mathrm{ROC})$ curve was used to evaluate the diagnostic value of miRNAs.

Results: Small RNA-Seq analysis showed that the Q30 value of HV and AS patients were higher than 95\% (Fig.1-A). The amount of miRNA in HV and AS patients were (509.667 \pm 77.501$)$ and (632.000 \pm 43.555$) .80$ up-regulated and 19 down-regulated exosomal miRNAs were identified in AS patients, compared with HV ( $\mid \log 2$ Ratio $>1, P<0.05$ ) (Fig.1-B-C). The target genes of the 34 highly-expressed miRNAs from the 99 differently-expressed miRNAs were 7869 , and the main function of these target genes are involved in the regulation of endocytosis and protein modification process analyzed by GO and KEGG. The qRT-PCR results indicated that the expression level of miRNA21-5P and miRNA423-5P in AS patients were $(2.940 \pm 1.572)$ and $(2.520 \pm 1.401)$ times higher than that of HV (Fig.1-D-E). ROC curve analysis showed that miRNA21-5P and miRNA423-5P had significant diagnostic value for AS with the AUC of 0.890 (CI95\%: 0.723-1.057) and 0.835 (CI95\%: 0.621-1.039) respectively (Fig.1-F).
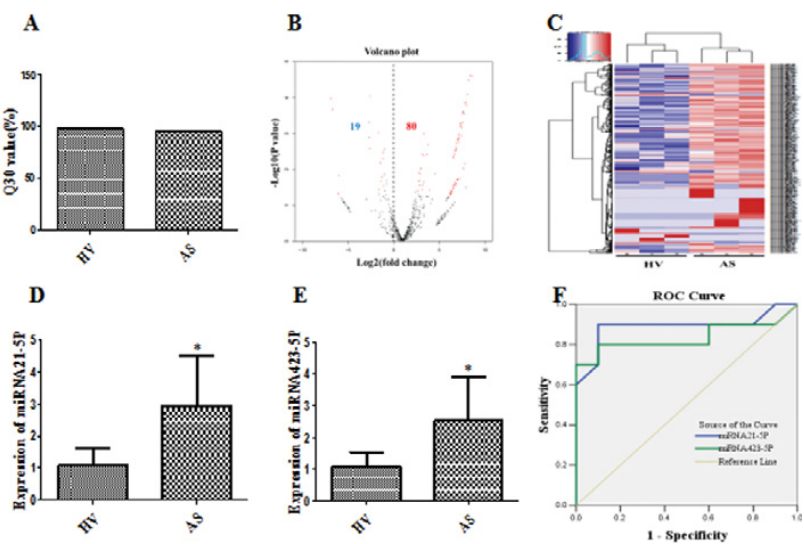

Fig.l Altered miRNAs profiles in plasma-derived exosome of AS by small RNA-Seq Analysis A: The Q30 value of each group. B-C: The differentially-expressed miRNAs. D-E: gRT-PCR was used to confirm the level of miRNA21-5P and miRNA423-5P.F: ROC cure was used to evaluate the tiagnostic value of miRNA21-5P and miRNA423-5P. $P<0.05$ vs HV.

Conclusions: The miRNAs profiles in plasma-derived exosome of AS patients are significant different from HV. miRNA21-5P and miRNA423-5P are higher expressed in AS patients. Thus, plasma-derived exosomal miRNAs might be reliable biomarkers to identify AS.

References:

[1] EI MA. Extra-articular manifestations of ankylosing spondylitis: prevalence, characteristics and therapeutic implications. Eur J Intern Med, 2011, 22(6):554-60.

Disclosure of Interest: None declared

DOI: 10.1136/annrheumdis-2017-eular.5637

\section{THU0011 IMMUNE SIGNAL 2 CHECKPOINT MOLECULE EXPRESSION IN RHEUMATOID ARTHRITIS DISEASE PROGRESSION}

A.M. Walsh ${ }^{1}$, M. Canavan ${ }^{2}$, Y. Guo ${ }^{1}$, T. McGarry ${ }^{2}$, X. Yin ${ }^{1}$, M.D. Wechalekar ${ }^{3}$, M.D. Smith ${ }^{3}$, S.M. Proudman ${ }^{4}$, C. Orr ${ }^{5}$, S. Kelly ${ }^{6}$, C. Pitzalis ${ }^{6}$, D.J. Veale ${ }^{5}$, U. Fearon ${ }^{2}$, S. Nagpal ${ }^{1}$. ' Janssen R\&d, Spring House, United States;

${ }^{2}$ Molecular Rheumatology, Trinity Biomedical Sciences Institute, Trinity College Dublin, Dublin, Ireland; ${ }^{3}$ Flinders University; ${ }^{4}$ Royal Adelaide Hospital and Discipline of Medicine, University of Adelaide, Adelaide, Australia; ${ }^{5}$ St. Vincent's University Hospital, Dublin, Ireland; ${ }^{6}$ Queen Mary University of London, London, United Kingdom

Background: Deep profiling of synovial tissue samples from rheumatoid arthritis (RA) patients may reveal the molecular underpinnings of phases of RA progression and provide new therapeutic targets to intervene earlier in disease pathogenesis. Objectives: We sought to identify the molecular pathways expressed in different stages of disease (from seropositive subjects without clinically apparent synovitis to those with established disease) in synovial tissue compared to non-RA controls. Methods: Transcriptomics profiling was performed on RNA isolated from synovial tissue biopsies. Normal synovium was collected from subjects with knee pain and without diagnosis of OA or RA $(n=28)$. Arthralgia tissue was collected from ACPA-positive subjects without synovitis $(n=10)$. Early RA tissue was collected from patients recently diagnosed ( $<1$ year) with $R A(n=57)$. Established RA tissue was collected from ACPA-positive subjects with $>1$ year of disease duration $(n=95)$. Protein expression was confirmed on infiltrating immune cells from synovial biopsy cell suspensions by flow cytometry in separate RA subjects.

Results: Several pathways previously identified as important for RA pathogenesis (e.g., lymphocyte activation, osteoclast differentiation, NF-kappa B signaling) were enriched in differentially expressed genes in disease synovial biopsies compared to normal tissue samples. Interestingly, several genes known to function in $T$ cell activation as signal 2 co-stimulatory or co-inhibitory molecules were differentially expressed, even in arthralgia and early RA subjects. 66 of 81 known co-stimulatory or co-inhibitory genes profiled were differentially expressed (FDR $<5 \%$ and absolute fold-change $>2$ ) in disease samples from at least one cohort. The genes encoding co-stimulatory proteins that were increased compared to normal included CD28, CD4OLG, CD40 and ICOS. Interestingly, some of the genes encoding co-inhibitory proteins were increased (PDCD1/PD-1, CD274/PDL1, HAVCR2/TIM3, TIGIT, BTLA), whereas others showed decreased expression (C10orf54/VISTA and LAG3) compared to normal controls. We focused on CD28 expression, which is elevated in "pre-RA" arthralgia samples, proposing that anti-CD28 therapeutics could be candidates for RA disease prevention. By flow cytometry we demonstrated that a majority of CD4+ (>90\%) and CD8+ $(>60 \%) \mathrm{T}$ 